\title{
Atomic Force Microscopy of DNA Immobilized onto a Highly Oriented Pyrolytic Graphite Electrode Surface
}

\author{
Ana Maria Oliveira Brett* and Ana-Maria Chiorcea \\ Departamento de Química, Faculdade de Ciências e Tecnol ogia, Universidade de Coi mbra, \\ 3004-535 Coimbra, Portugal
}

Received December 20, 2002. In Final Form: February 5, 2003

\begin{abstract}
Magnetic AC mode atomic force mi croscopy (MAC M ode AFM) was used to characterize the process of adsorption of DNA on a highly oriented pyrolytic graphite (HOPG) electrode surface using different concentrations of DNA and adsorption procedures. AFM of DNA immobilized on the HOPG showed that both single-stranded DNA and double-stranded DNA mol ecules have the tendency to spontaneously selfassemble from solution onto the solid support and the process was very fast. DNA condensed on the substrate in a tight and well-spread two-dimensional lattice covering the entire surface uniformly. The interaction of DNA with the hydrophobic HOPG surface induced DNA superposition, overlapping, and intra- and intermolecular interactions. The application of a positive potential of $300 \mathrm{mV}$ (vs Ag wire) to the HOPG electrode during adsorption was studied. The applied potential considerably enhanced the robustness and stability to mechanical stress of theDNA films, through multiple el ectrostaticinteractions between thenegatively charged hydrophilic sugar - phosphate backbone and the positively charged carbon surface. The characteristics of the DNA films and theapparent height of thenetwork wires weredependent on the DNA concentration and the immobilization procedure. The DNA lattices were held together on the substrate surface only by noncoval ent interactions such as hydrogen bonding, base stacking, el ectrostatic, van der Waals, and hydrophobic interactions.
\end{abstract}

\section{Introduction}

Great progress was madein devel oping small, portable, and viableDNA-based biosensors, which are of enormous interest duetotheir numerous potential applications. DNA biosensors are integrated receptor-transducer devices that use DNA as a biological recognition agent to detect chemical compounds, usually by electrical, thermal, or optical signal transduction. BecauseDNA is a stable, lowcost, and easily adaptable molecule, it is an extremely favorable medium for construction of new devices in nanotechnology and biosensor technology. ${ }^{1,2}$

DNA-electrochemical transducers have received particular attention due to advantages offered by electrochemical techniques, such as rapid detection, great sensitivity, and low cost. Between thetransducers, carbon el ectrodes such as glassy carbon, carbon fibers, graphite, or carbon black exhibit several unique properties. The wide electrochemical potential window, particularly in the positive range, allows the sensitive electrochemical detection of oxidative damage caused to DNA by monitoring the appearance of oxidation peaks from DNA bases. ${ }^{3}$ Combining the characteristics of the DNA probes with the capacity of direct and label-free electrochemical detection represents an attractive solution in many different fields of application.

Electrochemical DNA biosensors already have been successfully used in a variety of applications such as rapid monitoring of pollutant agents or metals in the environment, investigation and evaluation of DNA-drug interaction mechanisms, detection of DNA base damage in clinical diagnosis, direct monitoring of the hybridization

* To whom correspondence should be addressed. Tel/Fax: +351-239-835295. E-mail: brett@ci.uc.pt. 248.

(1) Seeman, N. C. Annu. Rev. Biophys. Biomol . Struct. 1998, 27, 225-

(2) Storhoff, J . J .; Mirkin, C. A. Chem. Rev. 1999, 99, 1849-1862.

(3) Oliveira Brett, A. M.; Matysik, F.-M.J . Electroanal. Chem. 1997, 429, 95-99. process, or detection of specific DNA sequences in human, viral, and bacterial nucleic acids. ${ }^{4-9}$

The DNA-electrochemical biosensor consists of an electrochemical transducer (the electrode) with a nucleic acid fil m immobilized on its surface (the probe). The DNA immobilization procedure on the electrode surface is a very important aspect influencing the characteristics of the DNA probe, the sensor response and its performance; several DNA adsorption methods were used on different types of carbon el ectrodes. ${ }^{10}$

The study of the adsorption of DNA on the electrode surface is of fundamental interest, since different structural conformations adopted by DNA can lead to different interactions with small molecules, to changes in accessibility of drugs to the DNA grooves, or to different rates of hybridization. Thespecificinteraction of DNA molecules with the electrode surface, the conformations that DNA can adopt during immobilization, and the degree of surface coveragearestill not well understood despitetheextensive use of DNA biosensors. The formation and stability, in solution and after drying during storage, of the DN A film in a DNA biosensor depends on many factors, such as el ectrode characteristics, immobilization procedures, adsorption time, pretreatment conditions, and thetype and concentration of DNA used. A good understanding of all

(4) Palecek, E. Talanta 2002, 56, 809-819.

(5) Palecek, E.; Fojta, M.; Tomschik, M.; Wang, J . 1998, 13, 621628.

(6) Oliveira Brett, A. M.; Serrano, S. H. P.; Piedade, J. A. P. Electrochemistry of DNA. In Applications of KineticM odel ling; Compton R. G., Ed.; ComprehensiveChemical Kinetics, Vol. 37; Elsevier: Oxford, U.K., 1999; Chapter 3, pp 91-119.

(7) Mascini, M.; Palchetti, I.; Marrazza, G. Fresenius'] . Anal . Chem. 2001, 369, 15-22.

(8) Oliveira Brett, A. M.; Silva, L. A. Anal. Bioanal. Chem. 2002, $373,717-723$

(9) Oliveira-Brett, A. M.; Vivan, M.; Fernandes, I . R.; Piedade, J . A. P. Talanta 2002, 56, 959-970.

(10) Pividori, M. I.; Merkoçi, A.; Alegret, S. Biosens. Bioel ectron. 2000, $15,291-303$ 
these factors is essential for improvement of existing sensors and development of new ones.

Atomic force microscopy (AFM) can give important information concerning the adsorption, nucleation, and growth of biological compounds at solid surfaces. AFM can visualizeindividual DNA molecules and DNA-protein complexes immobilized onto mica or silicon in air and in solution, with extraor dinary resolution and accuracy. ${ }^{11-15}$ Images of DNA on conducting substrates have been obtained mainly on gold via thiol modification of DNA. ${ }^{16}$ The highly oriented pyrolytic graphite(HOPG) surfaceas a substrate for DNA imaging has been the subject of scanning tunneling microscopy studies. ${ }^{17,18}$ DNA networks formed by specific oligomer sequences polyd $(A-T)_{2}$ and polyd $(G-C)_{2}$ at the surface of HOPG havebeen reported. ${ }^{19}$ However, DNA molecules have been found not to bind strongly enough to the conducting substrates required in electrochemical experiments and the AFM tip tends to remove the molecules attached to the surface.

Magnetic AC mode (MAC Mode) AFM imaging allows the investigation of molecules which are loosely attached to the substrate, especially in the case of conducting surfaces of electrochemical transducers. MAC M ode uses a solenoid placed under thesampleto causea magnetically coated AFM cantilever to oscillate near its resonant frequency. As it scans the sample, the AFM tip oscillates and touches the sample surface only at the bottom of this oscillation. Becausethereis no need to drivethecantilever holder, cantilever chip, or solution as in tapping mode, thecontrol of thecantilever increases considerably, which enables operation at smaller oscillation amplitudes even in air, the lateral forces being better eliminated.

In this paper, MAC M odeAFM was used to characterize the surface morphology of a DNA-electrochemical biosensor obtained by different immobilization procedures of calf-thymus double-stranded DNA (dsDNA) and singlestranded DNA (sSDNA) on a HOPG electrode surface.

\section{Experimental Section}

Materials. Calf-thymus dsDNA (sodium salt, type I) and ssDNA were purchased from Sigma-Aldrich Química, Madrid, Spain, and were used without further purification.

The electrolyte used was $\mathrm{pH} 7.0,0.1 \mathrm{M}$ phosphate buffer solution and was prepared using analytical grade reagents and purified water from a Millipore Milli-Q system (conductivity < $0.1 \mu \mathrm{S} \mathrm{cm}^{-1}$ ). Solutions of different concentrations were obtained by direct dilution of the appropriatevolume in phosphate buffer.

$\mathrm{HOPG}$, grade $Z Y H$, of rectangular shape with $15 \times 15 \times 2 \mathrm{~mm}$ dimensions, from Advanced Ceramics Co., U.K., was used throughout this study as a substrate. The HOPG was freshly cleaved with adhesive tape prior to each experiment and was imaged by contact modeAFM in order to establish its cleanliness.

Voltammetric experiments were carried out in a one-compartment Teflon cell of approximately $12.5 \mathrm{~mm}$ internal diameter

(11) Hansma, H. G.; Vesenka, J .; Siegerist, C.; Kelderman, G.; Morrett, H.; Sinsheimer, R. L.; Elings, V.; Bustamante, C.; Hansma, P. $\mathrm{K}$. Science 1992, 256, 1180-1184.

(12) Hansma, H. G.; Revenko, I.; Kim, K.; Laney, D. E. NucleicAcids Res. 1996, 24 (4), 713-720.

(13) U chihashi, T.; Tanigawa, M.; Ashino, M.; Sugawara, Y.; Yokoyama, K.; Morita, S.; I shikawa, M. Langmuir 2000, 16, 1349-1353.

(14) Hoyt, P. R.; Doktycz, M.J .; Modrich, P.; Warmack, R.J .; Allison, D. P. Ultramicroscopy 2000, 82, 237-244.

(15) Sanchez-Sevilla, A.; Thimonier, J .; Marilley, M.; Rocca-Serra, J .; Barbet, J . Ultramicroscopy 2002, 92, 151-158.

(16) Medalia, O.; Englander, J .; Guckenberger, R.; Sperling, J . Ultrami croscopy 2002, 90, 103-112.

(17) Clemmer, C. R.; Beebe, T. P. Science 1991, 251, 640-642.

(18) Clemmer, C. R.; Beebe, T. P. Scanning Microsc. 1992, 6, 319333.

(19) Tanaka, S.; Maeda, Y.; Cai, L. T.; Tabata, H.; Kawai, T. J pn. J Appl. Phys. 2001, 40, 4217-4220. holding the HOPG sample, the working electrode, on the base. A Pt wirecounter el ectrodeand a silver wireas a quasi-reference electrode were placed in the cell, dipping approximately $5 \mathrm{~mm}$ into the solution. Electrochemical control was done with a PalmSens potentiostat, running with PalmScan version 1.11, from Palm Instruments BV, The Netherlands.

DNA Sample Preparation. For DNA samples prepared by free adsorption, $100 \mu \mathrm{L}$ samples of different concentrations of DNA solutions were deposited onto freshly cleaved HOPG surfaces and incubated for $3 \mathrm{~min}$. The excess of DNA was gently cleaned with a jet of Millipore M illi-Q water, and theHOPG with adsorbed DNA was then dried with nitrogen.

For DNA samples prepared by el ectrochemical deposition, 500 $\mu \mathrm{L}$ of the desired DNA solution was placed in theelectrochemical cell holding the HOPG working electrode on the base. A positive potential of $300 \mathrm{mV}$ (vs Ag wire) was appl ied to the electrodefor $3 \mathrm{~min}$. The HOPG with adsorbed DNA was rinsed with a jet of Millipore Milli-Q water and dried with nitrogen.

Atomic F orce Microscopy. AF M was performed with a Pico SPM controlled by a MAC Mode module and interfaced with a PicoScan controller from Molecular I maging Corp., Tempe, AZ. All the AFM experiments were performed with a CS AFM S scanner with thescan range $6 \mu \mathrm{min} x-y$ and $2 \mu \mathrm{min} z$ (Molecular Imaging Corp.). Silicon type II MAClevers of $225 \mu \mathrm{m}$ length, 2.8 $\mathrm{N} / \mathrm{m}$ spring constant, and $60-90 \mathrm{kHz}$ resonant frequencies (Molecular I maging Corp.) were used in MAC M odeAFM. Silicon nitride NanoProbes V-shaped cantilevers of $100 \mu \mathrm{m}$ length and $0.58 \mathrm{~N} / \mathrm{m}$ spring constant were used for contact AFM in air.

All images were taken at room temperature, with scan rates of 1.0-1.3 lines $\mathrm{s}^{-1}$. The images were processed by flattening in order to remove the background slope, and the contrast and brightness were adjusted. All images were visualized in three dimensions using the Scanning Probe Image Processor, SPIP, version 2.3011, Image Metrology ApS, Denmark.

Statistics. Section analysis over DNA molecules and films was performed with PicoScan software version 4.19, Molecular Imaging Corp. The mean values of the heights were calculated using 50-100 measurements over different scanned images. Origin version 6.0 from Microcal Software, Inc., Northampton, $\mathrm{MA}$, was used to calculate standard deviation and all the experimental height/thickness distribution graphs.

\section{Results and Discussion}

Glassy carbon has been usually used to prepare DNAelectrochemical biosensors, but the rough and complex surfaces of these electrodes are not suitable for AFM surface characterization. For AF M studies, it is necessary to have an atomically flat substrate to clearly resolve the mol ecular adsorbed layer. Glassy carbon presents a rootmean-square (rms) roughness of $2.10 \mathrm{~nm}$ whiletheHOPG electrode surface presents a rms roughness of less than $0.06 \mathrm{~nm}$, as calculated from typical $1000 \mathrm{~nm} \times 1000 \mathrm{~nm}$ scan size contact AFM images in air of the glassy carbon and HOPG. Therefore, the atomically smooth HOPG el ectrode was used to study DNA immobilization by free adsorption and by applying a potential of $+300 \mathrm{mV}$ to the electrode during adsorption.

Immobilization of dsDNA and ssDNA on HOPG by $\mathrm{F}$ reeAdsorption. An electrochemical DNA bi osensor is very easy to prepare and does not require DNA or electrode surface modification as it can be obtained by freeadsorption of calf-thymus DNA on thecarbon electrode surface. This means that the characterization of the surface morphology of the biosensor is of the utmost importance. The adsorption time of $3 \mathrm{~min}$ has been used for preparing el ectrochemical DNA el ectrodes, sothis was the deposition time of interest to study the adsorption on the el ectrode surface. However, both free and controlled adsorption were performed for different deposition times ( $30 \mathrm{~s}, 3 \mathrm{~min}, 10 \mathrm{~min}$, and $25 \mathrm{~min}$ ). It was found that 30 $\mathrm{s}$ was insufficient, but above $3 \mathrm{~min}$ the formation of a featureless multilayer occurred. 

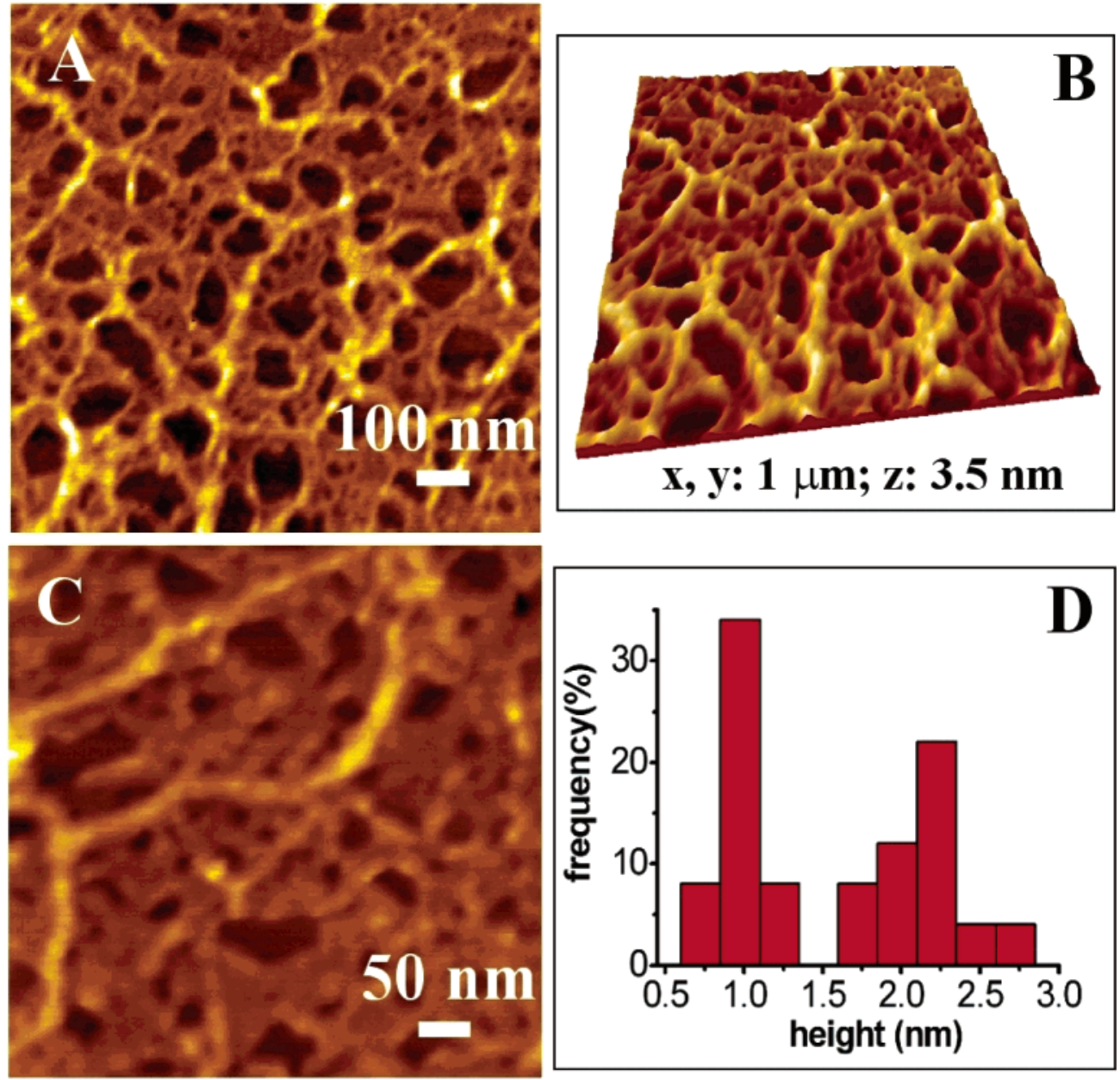

Figure 1. $(A, C)$ MAC Mode AFM topographical images in air of the DNA biosensor surface prepared by 3 min of free adsorption onto HOPG from a $60 \mu \mathrm{g} / \mathrm{mL}$ dsDNA in $\mathrm{pH}$ 7.0, $0.1 \mathrm{M}$ phosphate buffer el ectrolyte solution. (B) Three-dimensional representation of image A. (D) Histogram of the DNA film thickness generated with the values measured by section analysis inside the images.

Since the surface of the HOPG electrode presents hydrophobic characteristics and DNA is a highly charged, hydrophilic molecule, the capacity of spontaneous interaction of DNA with the HOPG surface should bereduced. H owever, DNA adsorbed freely at the HOPG surface, and MAC Mode AFM images in air revealed a good coverage of thesurfacein a film with theaspect of a two-dimensional network that will be described below.

dsDNA Free Adsorption. The HOPG electrode was modified by a thin film of dsDNA obtained by free adsorption of different concentrations of dsDNA using the procedure described in the Experimental Section.

Adsorption ontoHOPG from a $60 \mu \mathrm{g} / \mathrm{mL}$ dsDNA solution showed that the dsDNA molecules were freely adsorbed at the surface and were self-organized in a film with the aspect of a very tight and well-spread two-dimensional network, Figure 1 . The dsDNA network film has many holes, the dark regions in the images, leading to exposed HOPG surface at the bottom of the holes.

The height contour along the dsDNA network was slightly irregular. Section analysis performed inside various images permitted the construction of the height distribution graph in Figure 1C. Two typical heights of the network were present in the images: $0.99 \pm 0.25 \mathrm{~nm}$ and $2.15 \pm 0.25 \mathrm{~nm}$.

When the concentration of the dsDNA was lowered, it was observed that the coverage of the electrode surface decreased. ThedsDNA film becameless compact, and more extended areas of the HOPG surface were exposed. In
Figure 2A is presented a typical topographical image of the dsDNA films obtained from a $10 \mu \mathrm{g} / \mathrm{mL}$ dsDNA solution, and the average height over several regions of the film thickness was determined to be $1.10 \pm 0.20 \mathrm{~nm}$.

The initiation of network formation could be followed using $5 \mu \mathrm{g} / \mathrm{mL}$ dsDNA, Figure 2B. The dsDNA network loops were formed by aggregation of portions of single dsDNA molecules. In scans over different regions of the HOPG substrate, coiled as well as parts of straight dsDNA molecules with heights of $0.77 \pm 0.20 \mathrm{~nm}$ coul d beobserved.

The presence of salts in solution can induce lateral aggregation of the molecules. ${ }^{20}$ To assess the role of ions, the free adsorption of dsDNA using a solution of $1 \mu \mathrm{g} / \mathrm{mL}$ dsDNA in water was investigated. Themolecules appeared relaxed and more extended on theHOPG surface without the presence of the phosphate buffer ions in solution, Figure 3A.

The dsDNA attached to the HOPG surface is highly dehydrated and adopts the A-form with approximately $2.6 \mathrm{~nm}$ double-helix diameter. ${ }^{20}$ The measured full width at half-maximum height (fwhm) of dsDNA measured from the network loops and the straight DNA portions was approximately $10-25 \mathrm{~nm}$, which overestimates the helix diameter due to the convolution effect of the tip radius. The true representation of the dsDNA diameter is given by the height measurements, which are not limited by the

(20) Saenger, W. Principles of Nucleic Acid Structure; Cantor, Ch. R., Ed.; Springer Advanced Texts in Chemistry; Springer-Verlag: New York, 1984. 

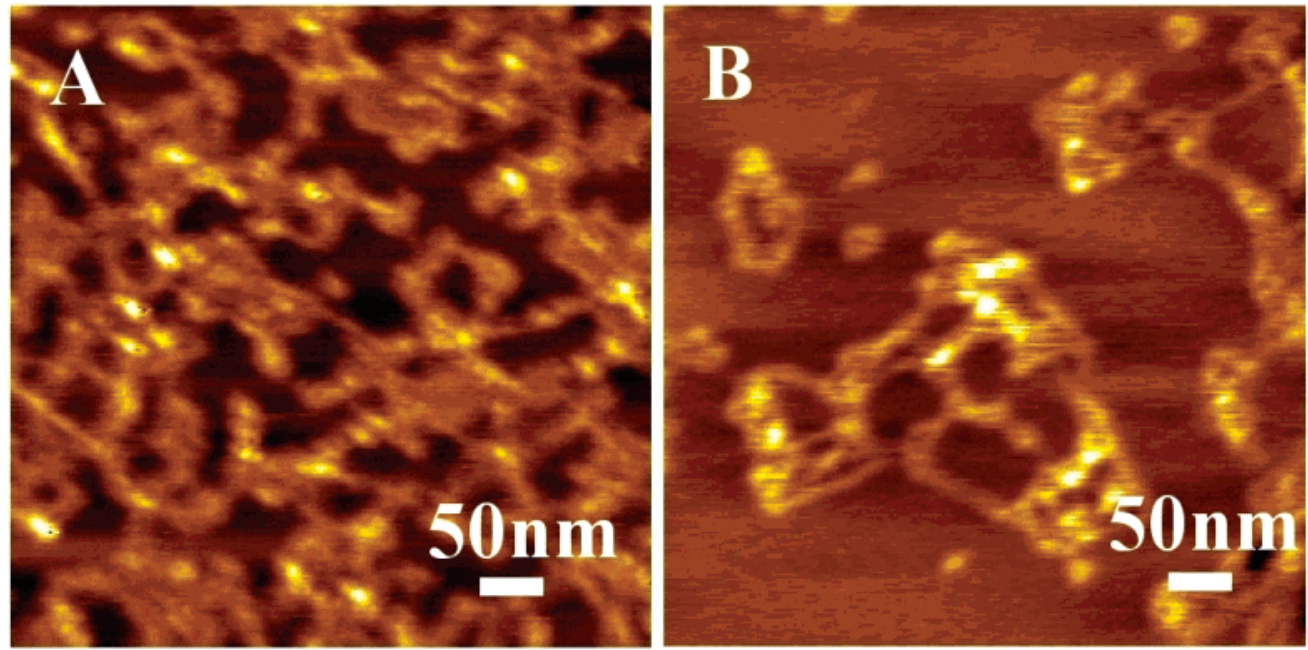

Figure 2. MAC Mode AFM topographical images in air of the DNA biosensor surface prepared by 3 min of free adsorption onto HOPG from (A) $10 \mu \mathrm{g} / \mathrm{mL}$ and (B) $5 \mu \mathrm{g} / \mathrm{mL}$ dsDNA in $\mathrm{pH} 7.0,0.1 \mathrm{M}$ phosphate buffer electrolyte solution.
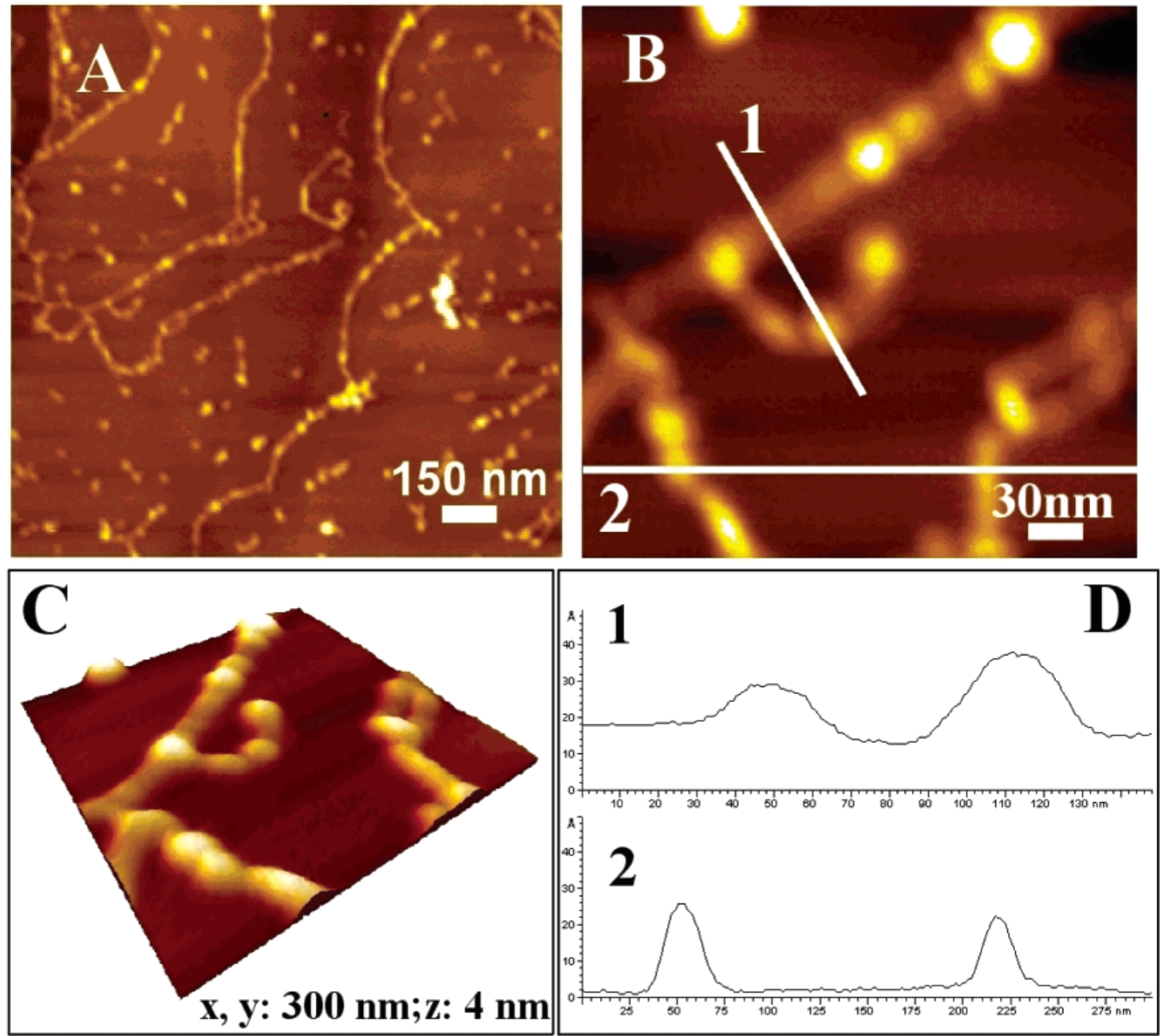

Figure 3. $(A, B)$ MAC Mode AFM topographical images in air of the DNA biosensor surface prepared by 3 min of free adsorption onto HOPG from a $1 \mu \mathrm{g} / \mathrm{mL}$ dsDNA sol ution in Milli-Q water. (C) Three-dimensional representation of image B. (D) Cross-section profiles through white lines 1 and 2 in image $B$ ).

tip radius. In AFM studies in air, the reported heights for dsDN A attached to a solid substrate vary from 0.5 to 1.9 $\mathrm{nm}^{12}$ and a height of $0.70 \pm 0.20 \mathrm{~nm}$ was obtained for dsDNA freely adsorbed onto HOPG. The difference between the true dsDNA height and the measured one is dueto elastic deformations caused on dsDNA by the AFM tip. The large fwhm of dsDNA was not only an artifact caused by the size and shape of the tip, but also an effect of dsDNA broadening as a consequence of dsDNA deformation by the tip.

Theaverage dsDNA height with its standard deviation measured by section analysis over portions of straight dsDNA molecules in the images obtained from $1 \mu \mathrm{g} / \mathrm{mL}$ dsDNA in water was $1.00 \pm 0.20 \mathrm{~nm}$. 

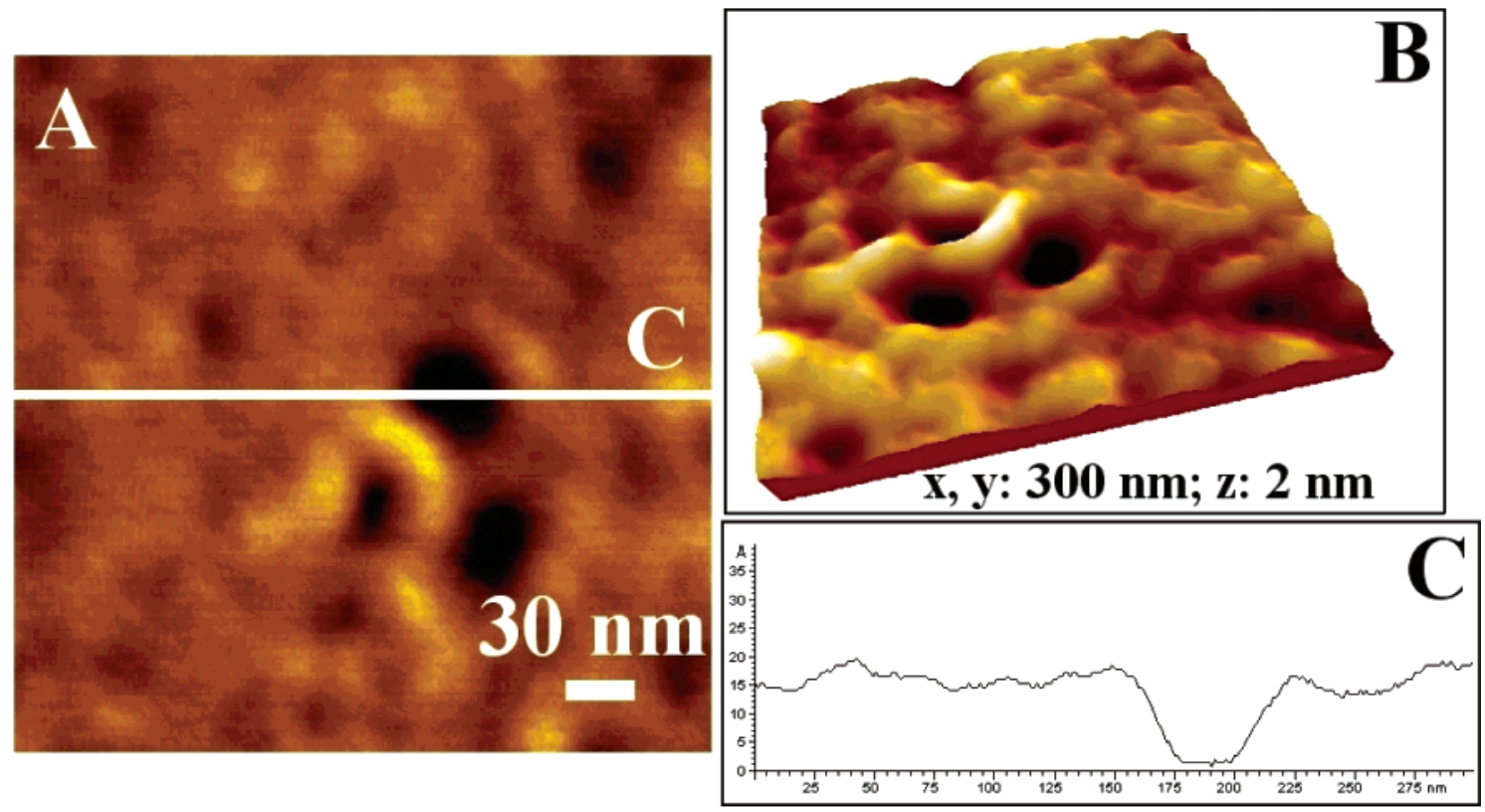

Figure 4. (A) MAC Mode AFM topographical image in air of the DNA biosensor surface prepared by 3 min of free adsorption onto HOPG from a $60 \mu \mathrm{g} / \mathrm{mL}$ ssDNA in pH 7.0, $0.1 \mathrm{M}$ phosphate buffer electrolyte solution. (B) Three-dimensional representation of image $A$. (C) Cross-section profile through the white line in image $A$.

The diameter of a single dsDNA molecule as visualized by MAC Mode AFM was compared with the thickness of the dsDNA films obtained at higher dsDNA concentrations. It can be concluded that theheight of approximately $0.8 \mathrm{~nm}$ for straight portions of dsDNA molecules and lattices obtained from the solutions with high concentrations is associated with A-DNA formed after the strong dehydration of the dried adsorbed molecules. The $2.15 \pm$ $0.25 \mathrm{~nm}$ thickness of the network layer observed when the deposition was made with high concentrations of dsDNA solution may be associated with more complex structures, dsDNA aggregation phenomena, and superposed dsDNA molecules.

The existence of a continuous dissociation-association of the bases of thedsDNA extremities exposesporadically the hydrophobic core of the DNA helix. ${ }^{20}$ The affinity of the hydrophobic bases to interact with the hydrophobic surface of theHOPG stabilizes thedsDNA at thesurface. ${ }^{21}$ When immobilization was performed from $1 \mu \mathrm{g} / \mathrm{mL}$ dsDNA dissolved in water, it was noticed that the dsDNA displayed spherical aggregates along its length (see the $300 \mathrm{~nm} \times 300 \mathrm{~nm}$ scan size image in Figure 3B), with sizes between 1.5 and $4.1 \mathrm{~nm}$ (Figure $3 \mathrm{C}$ ). This indicates that the presence of ions is not the only reason for aggregation. Consequently, it can be concluded that the hydrophobic HOPG surface induces aggregation of long dsDNA, even in the absence of the buffer solution. The ability of HOPG and other hydrophobic polymericsurfaces to adsorb and aggregate dsDNA was previously observed. $19,21,22$ Due to the interaction of dsDNA with the HOPG substrate, themolecules overlap and superimpose, interacting through "sticky-ended" cohesi ons, ${ }^{1}$ leading to conformation changes, DNA-DNA interactions, and formation of complex lattices. However, from the present data it is not possible to determine exactly which type of interaction occurs at the HOPG surface or the internal conformation transitions that are induced by the surface alone.

(21) Allemand, J .-F.; Bensimon, D.; J ullien, L.; Bensimon, A.; Croquette, V. Biophys. J. 1997, 73, 2064-2070.

(22) Gaillard, C.; Strauss, F. Am. Clin. Lab. 2001, 20 (2), 52-54.
ssDNA Free Adsorption. The next step in this study was to analyze the DNA biosensor surface prepared by free adsorption of ssDNA.

I mages of adsorption onto HOPG from a $60 \mu \mathrm{g} / \mathrm{mL}$ ssDNA solution showed a dry uniform thin film network covering the electrode al most completely and only a few pores, with a thickness of $0.98 \pm 0.40 \mathrm{~nm}$, Figure 4 . Adsorption from a $10 \mu \mathrm{g} / \mathrm{mL}$ ssDN A solution still showed a compact network.

A noncompact two-dimensional lattice self-assembled on the HOPG surfacewas observed using $5 \mu \mathrm{g} / \mathrm{mL}$ ssDNA, Figure 5. The network was composed of looped filaments that form pores, and large portions of uncovered HOPG surface. The histogram of the measured thickness of the ssDNA film gives information about the height distribution of the ssDNA loops, Figure 5C. The heterogeneity of the values indicates that portions of ssDNA could form the film as well as double-stranded parts resulting from hybridization of randomly complementary zones.

Comparison between dsDNA and ssDNA FreeAdsorption. This work shows that calf-thymus dsDNA forms networks at the HOPG surface. This capability of selfassembly into complex lattices is al so observed here for thefirst timewith ssDNA dueto hybridization of randomly complementary zones. The topography of the ssDNA modified surface suggests that ssDNA interacts and adsorbs strongly to the HOPG surface, when compared with dsDNA, for the same concentration of $5 \mu \mathrm{g} / \mathrm{mL}$, Figures $2 \mathrm{~B}$ and 5 . This can be explained because the sSDNA has the bases exposed to the solution, which facilitates interaction with thesubstrate. Thehydrophobic aromatic rings of the ssDNA bases attach by hydrophobic interactions to the hydrophobic carbon surface.

I mmobilization by free adsorption is the simplest way to attach nucleic acid to the surface of the transducers, but simple adsorption methodologies have big disadvantages. DNA often coils, aggregates, and moves under the scanning probe whileresting on a substratesurface. DNA self-assemblies involvea big number of weak interactions; consequently large-scal eDNA lattices are rather unstable and the nucleic acid may desorb easily from the surface 


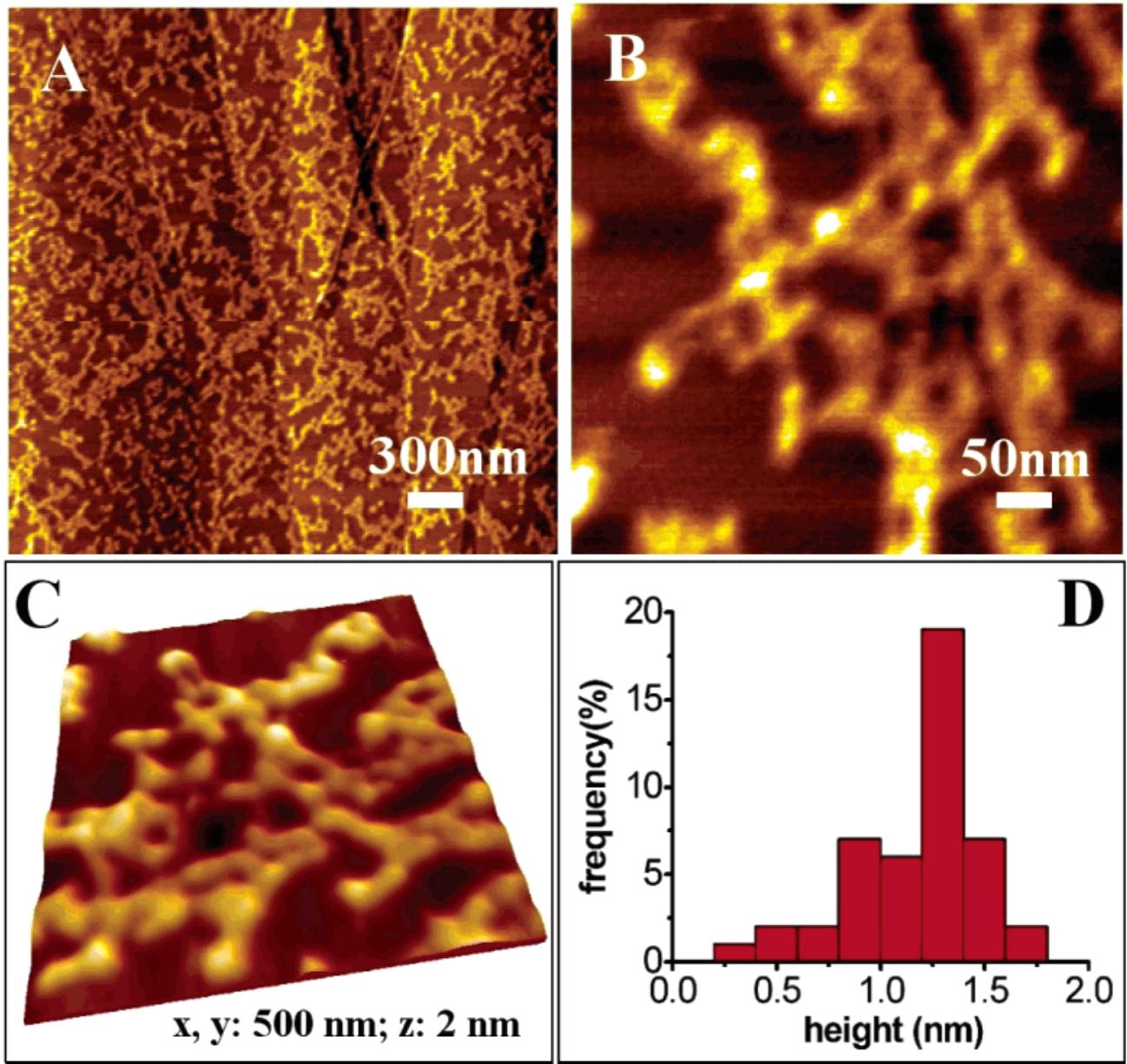

Figure 5. $(A, B)$ MAC Mode AFM topographical images in air of the DNA biosensor surface prepared by 3 min of free adsorption onto HOPG from a $5 \mu \mathrm{g} / \mathrm{mL}$ ssDNA in pH 7.0, $0.1 \mathrm{M}$ phosphate buffer electrolyte solution. (C) Three-dimensional representation of image B. (D) Histogram of the DNA film thickness generated with the values measured by section analysis inside the images.

in routine experimental conditions. ${ }^{19}$ It was observed during the experiments that the AFM tip could easily remove both single and double-stranded DNA films obtained by free adsorption; also due to the loose attachment to the surface the DNA molecules were much more easily compressed by the AFM loading forces.

Immobilization of dsDNA and ssDNA on HOPG under an Applied Potential. By application of a potential to the electrode, controlled adsorption of DNA onto the HOPG surface can be obtained. The effect of electrochemically assisted adsorption of DNA on the HOPG electrode surface was assessed, and the surface characteristics of the DNA biosensor were analyzed. The potential of $+300 \mathrm{mV}$ (vs Ag wire) is not sufficiently high to oxidize the DNA bases $^{3}$ and was proved to stabilize efficiently the DNA molecules on the surface. The HOPG electrode was immersed in different concentrations of ssDNA and dsDNA in the conditions described in the Experimental Section.

dsDNA Adsorption under an Applied Potential. MAC ModeAFM images in air of dsDNA on the HOPG electrode surface, prepared from a $60 \mu \mathrm{g} / \mathrm{mL}$ solution, showed a self-assembled network in a two-dimensional dsDNA lattice, Figure 6A, which spread uniformly over the scanner's maximum scan size, $7 \times 7 \mu \mathrm{m}^{2}$.

In MAC Mode AFM, there exists the possibility of recording the changes in the phase of the cantilever oscillation during the scan to obtain a second image, the phase image. Usually the phase image gives improved contrast with respect to the topographic image, helping to confirm the existence of features and conformations of molecules on the surface, ${ }^{23}$ especially using HOPG. ${ }^{17}$ Generally thechanges in the phase angleduringscanning are related to changes in material or surface properties, such as chemical composition, stiffness, or viscosity. F rom $300 \mathrm{~nm} \times 300 \mathrm{~nm}$ scan size MAC Mode AF M topography and phaseimages recor ded simultaneously, images $C$ and D of Figure 6, respectively, it was observed that thedsDNA lattice was formed by coiled fibers which join together in end-to-end aggregations, stabilizing them on the HOPG surface. The dsDNA condensates twisted together in large and rather flexible loops, leaving big parts of the HOPG surface uncovered. Using the phaseimagefrom MAC Mode $A F M$, it was noticed that changes in phase angle occur when the tip encountered the dsDNA fibers, which confirmed that the HOPG substrate was really observed under the DNA adsorbates.

The measured thickness along the network arms was highly irregular, with heights between 3.5 and $7.5 \mathrm{~nm}$, Figure6E. Thevalues weremuch higher than theexpected height of A-form DNA, meaning that several layers of dsDNA molecules were involved in the condensation process. This leads to the conclusion that the applied potential increased the attractive lateral interaction between adjacent dsDNA helices and caused spontaneous condensation of the dsDNA film in a complex network on the HOPG surface.

(23) Argaman, M.; Golan, R.; Thomson, N. H.; Hansma, H. G. Nucleic Acids Res. 1997, 25 (21), 4379-4384. 

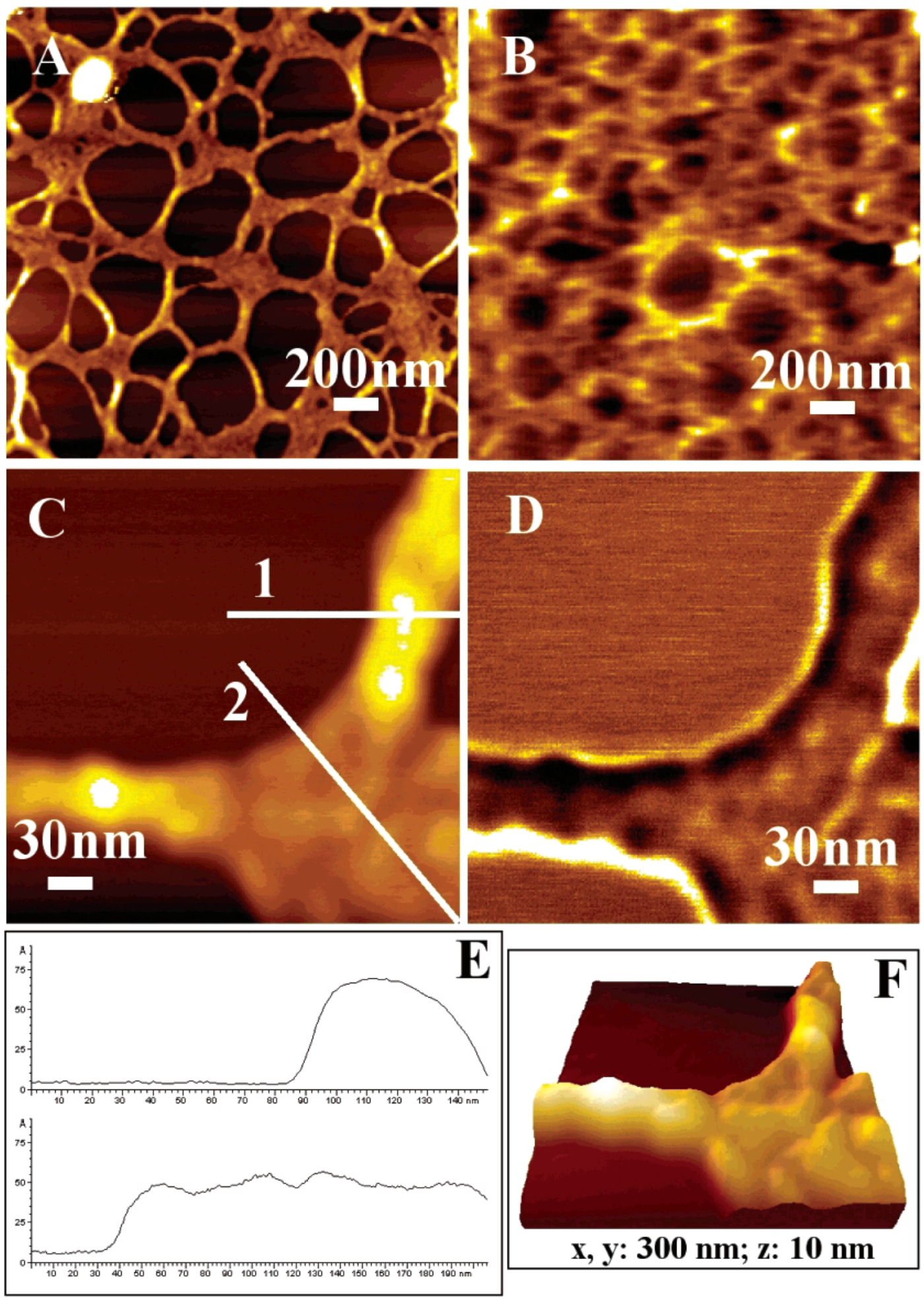

Figure 6. $(A, C)$ MAC Mode AFM topographical images in air of the DNA biosensor surface prepared by applying a deposition potential of $+300 \mathrm{mV}$ (vs Ag wire) for $3 \mathrm{~min}$ to the HOPG electrode immersed into a $60 \mu \mathrm{g} / \mathrm{mL}$ dsDNA pH 7.0, $0.1 \mathrm{M}$ phosphate buffer electrolytesolution. (B) Contact AFM image of the DNA film recorded in a pH 7.0, 0.1 M phosphate buffer electrol yte sol ution. (D) Phase image recorded simultaneously with the topographic image $C$. (E) Cross-section profiles through white lines 1 and 2 in image C. (F) Three-dimensional representation of image C.

Thestability of thedsDNA film was increased very much duetoelectrostaticinteraction with the positively charged HOPG el ectrode surface. An in situ contact AFM image of the DNA film after reimmersing the substrate in $\mathrm{pH}$ 7.0, $0.1 \mathrm{M}$ phosphate buffer solution is shown in Figure $6 \mathrm{~B}$. In this way, the cycle of drying and reimmersing the surface in buffer is similar to the real case of electrochemical experiments involving dsDNA-modified elec- trodes. The dsDNA adsorbed film was robust enough to resist the high forces applied in contact AFM. It was observed that the dsDNA network had thicker loops corresponding to hydrated fibers, although the dsDNA film still had holes that exposed the HOPG electrode surface.

The applied potential modifies the conformation of dsDNA on the electrode surface. The positively charged 

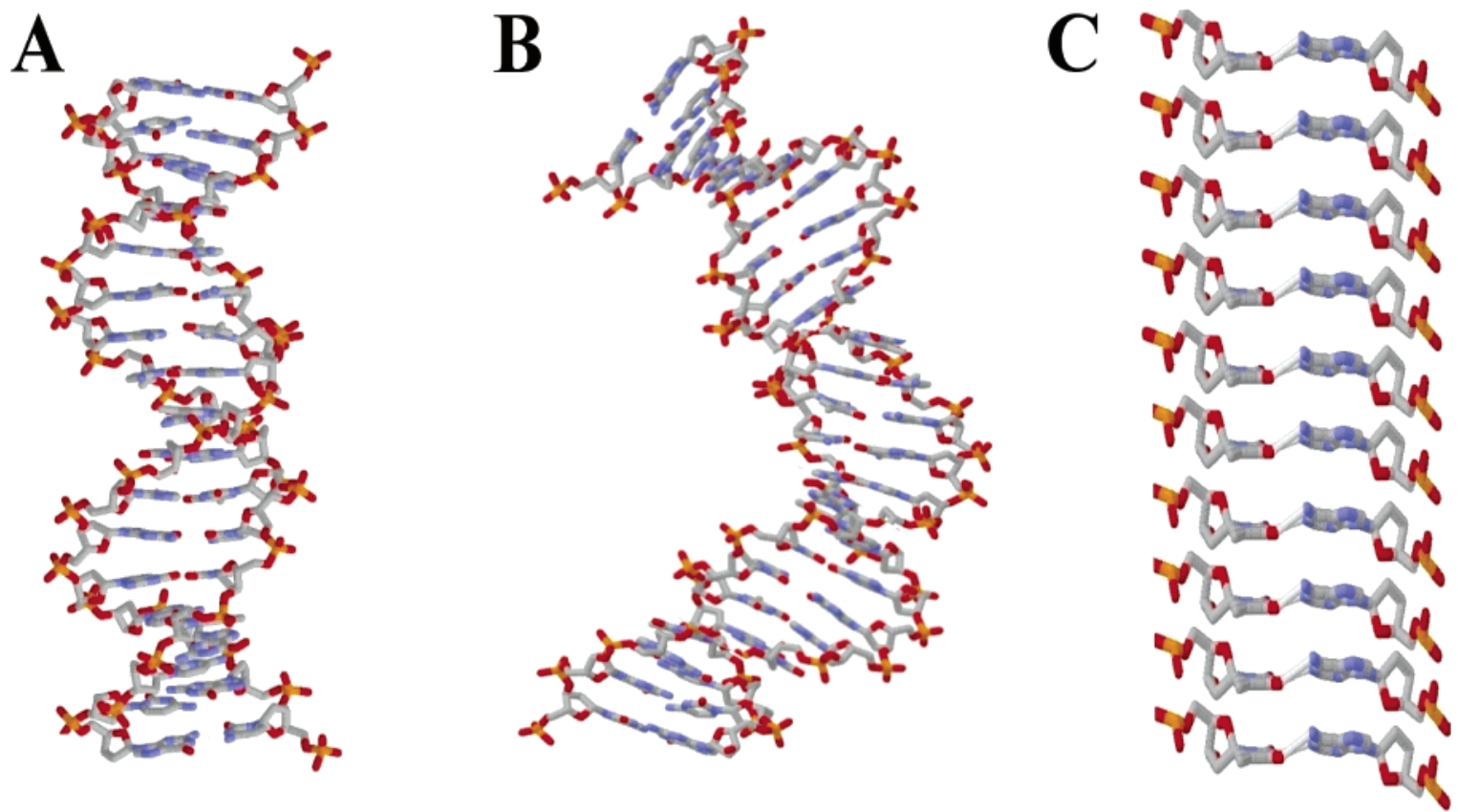

Figure 7. Models of schematic structural conformations of dsDNA at the HOPG electrode surface.
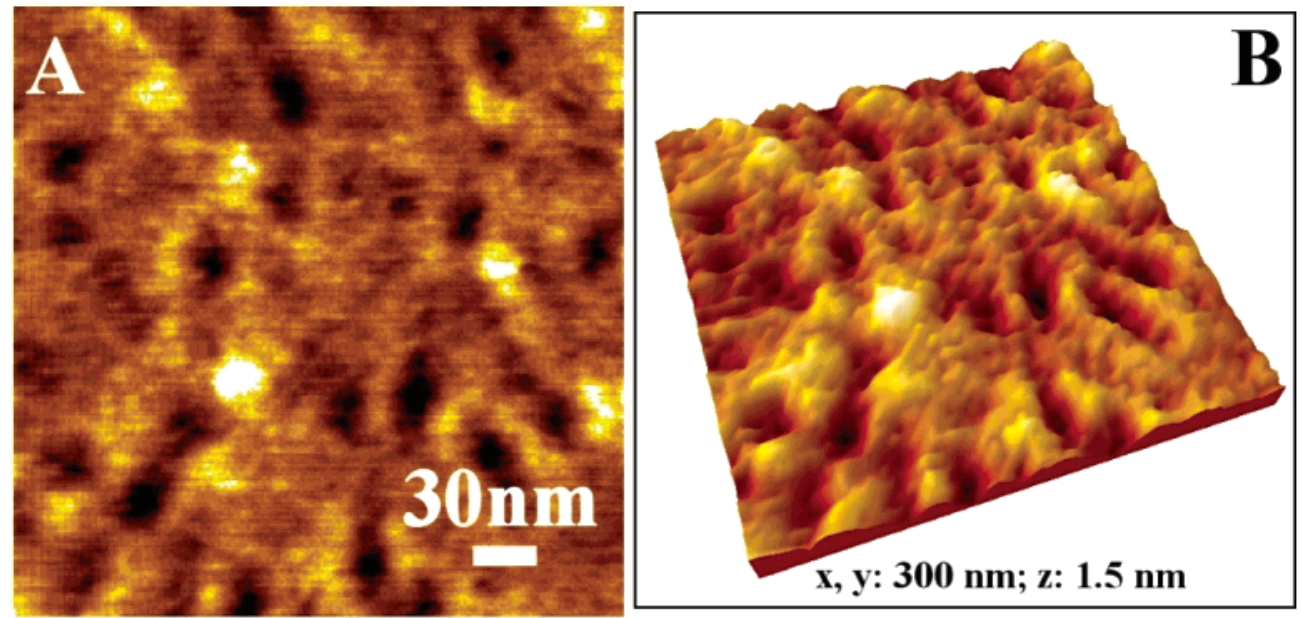

Figure 8. (A) MAC Mode AFM topographical images in air of the DNA biosensor surface prepared by applying a deposition potential of $+300 \mathrm{mV}$ (vs Ag wire) for $3 \mathrm{~min}$ to the electrode immersed into a $60 \mu \mathrm{g} / \mathrm{mL}$ ssDNA pH $7.0,0.1 \mathrm{M}$ phosphate buffer electrolyte solution. (B) Three-dimensional representation of image A.

HOPG substrate exerts electrostatic attraction on the nucleic acid molecules. DNA is a helical molecule, and in aqueous sol ution the negativel y charged phosphategroups of the dsDNA are outside the duplex, Figure 7A. Both dsDNA strands are equally attracted to the surface because the phosphate groups along the two strands are equivalent. The positively charged electrode neutralizes the negative phosphate charges only on one side of the DNA doublehelix. On theoppositeside, thecharge-charge repulsion is still present, which facilitates DNA bending and looping, ${ }^{20}$ Figure $7 \mathrm{~B}$, as is also observed in all the MAC Mode AFM images. This phenomenon is probably followed by structural rearrangement of the molecule. During reorientation and equilibration of the biopolymer on the surface, the helix is destabilized, that is, some phosphate groups detach from the charged electrode facilitating theelectrostatic binding on the HOPG surface of the phosphategroups from thesamestrand and leading to the formation of nonhelical DNA parts, Figure 7C. The phenomenon of formation of asymmetrical duplex structures was previously described. ${ }^{24}$ As a consequence, parts of the phosphate backbone of one strand lie down flat on thesurface, which may explain the differencein theheight along theduplex. The destabilization and local stretching of the DNA duplex may involve a significant loss of base stacking and hydrogen bonding. The DN A bases initially protected inside the helix appear more exposed to the solution and free to undergo intermolecular interactions by hydrogen bonding and base stacking with bases from other chains that bind nearly on the surface. End-to-end interactionsstabilizetogether themolecules. Closestrands intertwine with each other with the formation of complex compact structures.

ssDNA Adsorption under an Applied Potential. MAC ModeAFM images in air of SsDNA on the HOPG el ectrode surface also showed a uniform self-assembled network. The surface coverage with decreasing ssDNA concentration was monitored, the characteristics of the DNA film depending strongly on the solution concentration.

(24) Lemeshko, S. V.; Powdrill, T.; Belosludtsev, Y. Y.; Hogan, M. Nucleic Acids Res. 2001, 29 (14), 3051. 

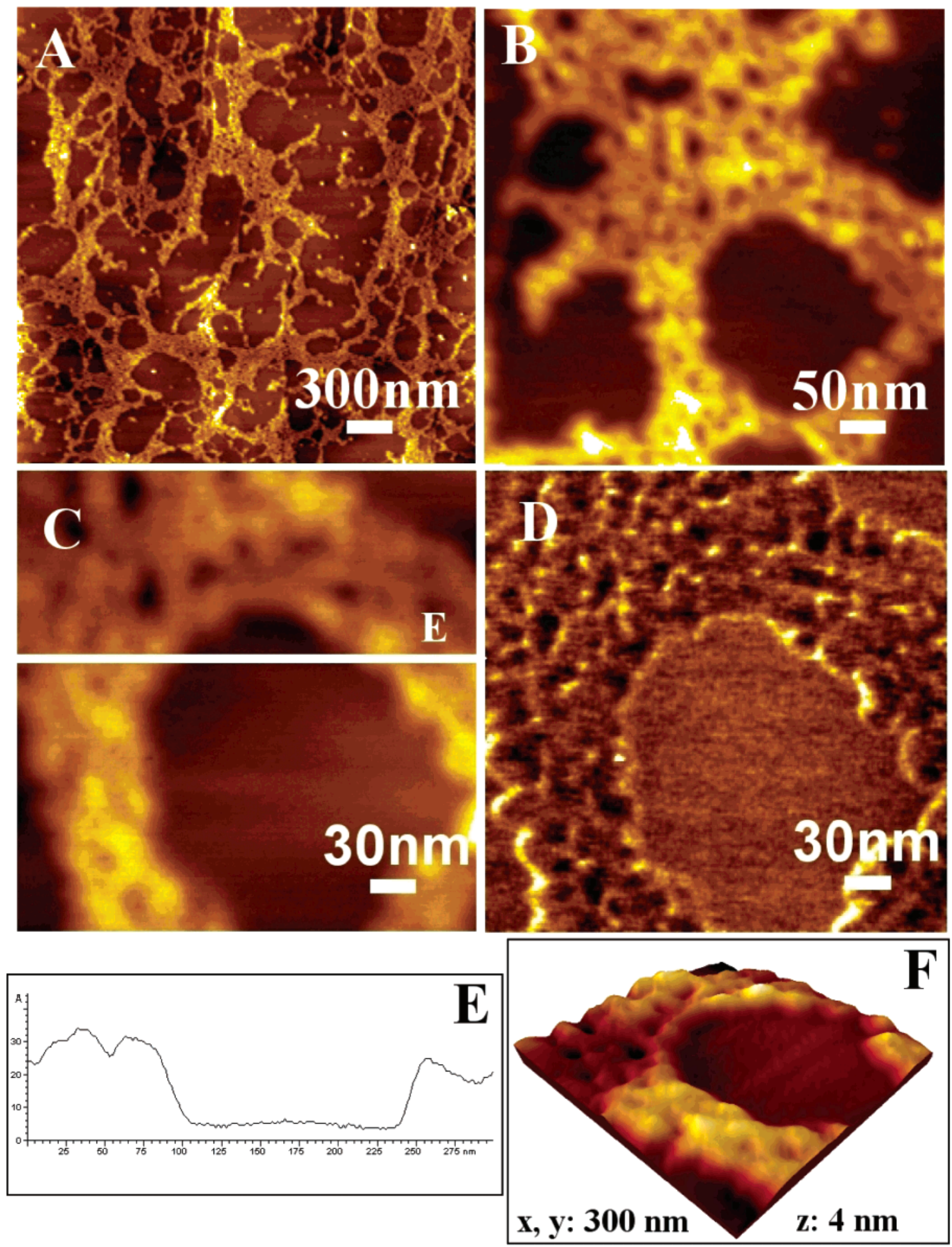

Figure 9. $(A-C)$ MAC Mode AFM topographical images in air of the DNA biosensor surface prepared by applying a deposition potential of $+300 \mathrm{mV}$ (vs Ag wire) for $3 \mathrm{~min}$ to the electrode immersed into a $5 \mu \mathrm{g} / \mathrm{mL}$ ssDNA pH 7.0, $0.1 \mathrm{M}$ phosphate buffer electrolyte solution. (D) Phase image recorded simul taneously with the topographic image C. (E) Cross-section profile through the white line in image C. (F) Three-dimensional representation of image C.

At high concentrations of $60 \mu \mathrm{g} / \mathrm{mL}$ ssDNA solutions, an almost completely two-dimensional network with few holes was covering the electrode, Figure 8.

A less densely packed network could be imaged all over the HOPG electrode surface using $5 \mu \mathrm{g} / \mathrm{mL}$ ssDNA solutions, Figure 9, and the SSDNA molecules demonstrated predisposition to condense together in extensive thin lattices covering big areas of the substrate. Between neighboring thin lattices, large zones of the HOPG were not cover ed at all. Thetopographical images, Figure9B,C, and the respective phaseimage (with enhanced contrast), Figure9D, show that thenetwork morphol ogy is composed by very tight loops with small pores that almost do not reveal theHOPG surface. Many of thessDNA holes in the layer were not big enough to leave the HOPG electrode surface exposed after possiblerehydration of thesubstrate. 
The measured thickness of the film was $2.37 \pm 0.4 \mathrm{~nm}$, Figure 9E, which suggests that more than onemonolayer of ssDNA molecules condensed together. This phenomenon may bedueto crossover molecules or to binding of stickyended linear strands while forming the lattice. It is al so very probable that many parts of the ssDNA molecules contain complementary bases leading to the formation of portions of dsDNA by hybridization events.

Comparison between dsDNA and ssDNA Adsorption under an Applied Potential. As in the case of dsDNA, the application of a potential of $+300 \mathrm{mV}$, versus Ag wire, enhanced the strength, robustness, and resistance to mechanical stress of the ssDNA film. Electrostatic interactions between the negative charges along the dsDNA and SSDNA phosphatebackboneand thepositively charged HOPG el ectrodesurfacewerevery strong, which increased stability of the molecules on the substrate. Consequently theadsorbed mol ecules wereless compressi ble by theAFM tip. Many mol ecules interact together by hydrogen bonding during equilibration on the substrate, and hydrophobic interactions and van der Waals forces also contribute to adsorption of DNA on the HOPG electrode.

\section{Conclusions}

Theresults indicatethat both dsDNA and ssDNA adsorb freely at the HOPG surface; the process of adsorption was shown to bevery fast, and themolecules havethetendency tospontaneously self-assemblefrom solution ontothesolid support, forming large-scale two-dimensional networks uniformly covering the entire HOPG substrate.
Theformation of a robust and stable DN A network onto HOPG was successfully achieved by electrochemically assisted adsorption. The stability of the adsorbed DNA layers after transfer intothebuffer solution is considerably enhanced when adsorption occurs under an applied potential. The characteristics of the DNA networks and the apparent height of the film depend on DNA concentration, form (ssDNA or dsDNA), and applied el ectricfield. The DNA lattices are held together at the HOPG surface only by noncovalent interactions such as hydrogen bonding, base stacking, electrostatic, van der Waals, and hydrophobic interactions.

MAC Mode AFM in an electrochemically controlled environment offers a significant potential for resolving the surface morphol ogical features of immobilized DNA and may contribute to the understanding of the mechanism of adsorption and the nature of DNA-electrode surface interaction, as well as to the improvement and development of DNA-electrochemical biosensors.

Acknowledgment. Financial support from Fundação para a Ciência eTecnol ogia (FCT), Ph.D. Grant PRAXIS XXI/BD/19728/99 (A.-M. C.), POCTI (cofinanced by the European Community Fund FEDER), ICEMS (Research Unit 103), and European Projects QLK3-200001311 and HPRN-CT-2002-00186 is gratefully acknowledged.

LA027047D 